Aktuelle Entwicklungen in der Therapie des multiplen Myeloms

\title{
Bortezomib: Hohe Ansprechqualität und verlängertes Überleben
}

\begin{abstract}
Bei Patienten mit rezidiviertem multiplen Myelom induziert der Proteasominhibitor Bortezomib $\left(V E L C A D E^{\circledR}\right)$ eine hohe Rate kompletter und fast kompletter Remissionen, die nach den vorliegenden Studiendaten mit einem längerfristigen klinischen Benefit assoziiert sind. Nach aktuellen Daten einer Subgruppen-Analyse der APEX-Studie können auch Patienten mit Nierenfunktionsstörungen von Bortezomib profitieren. Bemerkenswert sind darüber hinaus die positiven Effekte des Proteasominhibitors auf den Knochenstoffwechsel: Als einzige beim multiplen Myelom wirksame Substanz gibt es hier Hinweise auf eine Hemmung der gesteigerten Osteoklastenfunktion und Aktivierung der reduzierten Osteoblastenfunktion.
\end{abstract}

Die Zulassung von Bortezomib als Monotherapie bei Patienten mit multiplem Myelom ab dem ersten Rezidiv nach erfolgter oder nicht indizierter Stammzelltransplantation basiert auf den Ergebnissen der APEX (Assessment of Proteasome Inhibition for Extended Remissions)-Studie. In der randomisierten Phase-III-Studie induzierte Bortezomib $\left(1,3 \mathrm{mg} / \mathrm{m}^{2}\right.$, Tag 1, 4, 8, 11; q3w) bei Patienten mit median 2 Vortherapien im Vergleich zu hochdosiertem Dexamethason statistisch signifikant mehr komplette und fast komplette Remissionen $(\mathrm{CR} / \mathrm{nCR})$ und zeigte als erste Monosubstanz einen klinisch relevanten Überlebensvorteil. Die mediane Überlebensdauer betrug nach einem mittleren Follow-up von 22 Monaten im Bortezomib-Arm 29,8 Monate, gegenüber 23,7 Monaten im Kontrollarm [1].

Bereits der berichtete Überlebensvorteil von 6 Monaten stellt einen bemerkenswerten Fortschritt in der Therapie vorbehandelter Patienten mit rezidiviertem multiplen Myelom dar. Tatsächlich dürfte der Überlebenszeitgewinn durch den Proteasominhibitor aber noch größer sein; denn in der APEX-Studie wurden im Kontrollarm 62\% der Patienten bei Progress auf Bortezomib umgestellt, diese Ergebnisse entsprechend dem Studienprotokoll aber weiterhin der Dexamethason-Gruppe zugerechnet.

\section{Hohe Ansprechqualität prädiktiv für längerfristigen Benefit}

Als Surrogatmarker für einen längerfristigen Nutzen von Bortezomib identifizierte die Ar- beitsgruppe von Ruben Niesvizky das Erreichen einer $\mathrm{CR} / \mathrm{nCR}$ [2]. In der APEX-Studie erzielten von den Patienten im BortezomibArm $15 \%$ eine $\mathrm{CR} / \mathrm{nCR}$, bei $28 \%$ lag eine PR vor. In der SUMMIT-Studie, an der stark vorbehandelte Patienten mit median 6 Vortherapien teilnahmen, sprachen von den für eine Bortezomib-Response auswertbaren Patienten $10 \%$ mit einer $\mathrm{CR} / \mathrm{nCR}$ an, $18 \%$ zeigten eine partielle Remission (PR).

Das Erreichen einer $\mathrm{CR} / \mathrm{nCR}$ war in beiden Studien prädiktiv für einen längerfristigen klinischen Nutzen durch Bortezomib. In der APEX-Studie dauerte die behandlungsfreie Zeit (TFI), definiert als Intervall zwischen der letzten Bortezomib-Dosis und der 1. Dosis einer weiteren Therapie, bei Patienten mit $\mathrm{CR} / \mathrm{nCR}$ median 17,5 Monate und war damit mehr als doppelt so lang wie bei Patienten mit PR (6,7 Monate). Der Nutzen einer hohen Ansprechqualität zeigte sich auch in der medianen Zeit bis zur Progression, die bei Patienten mit einer durch Bortezomib induzierten $\mathrm{CR} / \mathrm{nCR}$ 12,2 Monate betrug, gegenüber 8,3 Monaten bei einer PR (Abb. 1). Die Ergebnisse der SUMMIT-Studie zeigen ebenfalls die Vorteile eines besseren Ansprechens deutlich: Die behandlungsfreie Zeit bei Patienten mit $\mathrm{CR} / \mathrm{nCR}$ war mit median 9,8 Monaten gegenüber 3,1 Monaten bei einer PR deutlich länger; die Zeit bis zur Progression betrug bei einer CR/nCR 16,4 Monate gegenüber 9,2 Monate bei einer PR. Die Auswirkungen der Ansprechqualität auf das Gesamtüberleben der Bortezomib-Responder konnten noch nicht abschließend analysiert werden, da nach einem Follow-up von 22 bzw. 23 Monaten das mediane Überleben bisher weder in der APEX-Studie noch in der SUMMIT-Studie erreicht wurde [2]. Die Ergebnisse dieser Analyse legen nahe, Patienten, die auf Bortezomib zunächst nur mit einer partiellen Remission ansprechen, für insgesamt bis zu 8 Zyklen weiter zu behandeln. Denn obwohl in der APEX-Studie die mediane Zeit bis zum Therapieansprechen mit 1,4 Monaten sehr kurz war und circa 50\% der Patienten das beste Therapieansprechen bereits nach 4 Zyklen erreichten $[1,3]$, «verbesserte sich das Ansprechen mit zunehmender Therapiedauer», wie Priv.Doz. Dr. med. Guido Kobbe, Düsseldorf, im Rahmen einer Pressekonferenz anlässlich des diesjährigen DGHO unterstrich.

\section{Nutzen von Bortezomib bei einge- schränkter Nierenfunktion}

Circa 30\% der Myelom-Patienten weisen zum Teil schwere Nierenfunktionsstörungen auf, die ein Indiz für eine fortgeschrittene Erkrankung und hohe Tumorlast darstellen und per se mit einer kürzeren Überlebensdauer assoziiert sind. Zusätzlich beeinträchtigt wird die Prognose oft dadurch, dass Zytostatika mit renaler Elimination bzw. Toxizität bei diesen Patienten niedriger dosiert werden müssen als bei nierengesunden Myelom-Patienten [4].

Für Bortezomib konnte in einer Dosiseskalationsstudie bei Patienten mit fortgeschrittenen malignen Erkrankungen und leichter bzw. moderater Niereninsuffizienz (Kreatinin-Clearence $\geq 20 \mathrm{ml} / \mathrm{min} / 1,73 \mathrm{~m}^{2}$ ) gezeigt werden, dass die Substanz in der empfohlenen Dosierung von $1,3 \mathrm{mg} / \mathrm{m}^{2}$ an den Tagen 4, 8, 11, q3w auch bei eingeschränkter Kreatinin-Clearance gut verträglich ist, wenn die Patienten sorgfältig überwacht werden [5]. Die Wirksamkeit und Verträglichkeit von Bortezomib bei fortgeschrittener Niereninsuffizi-

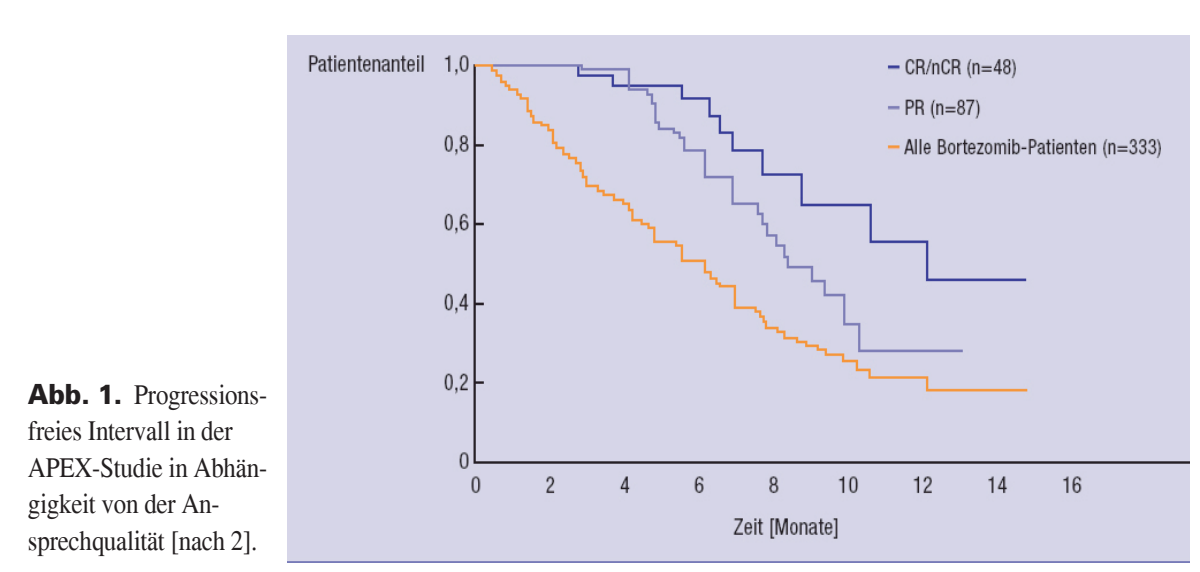




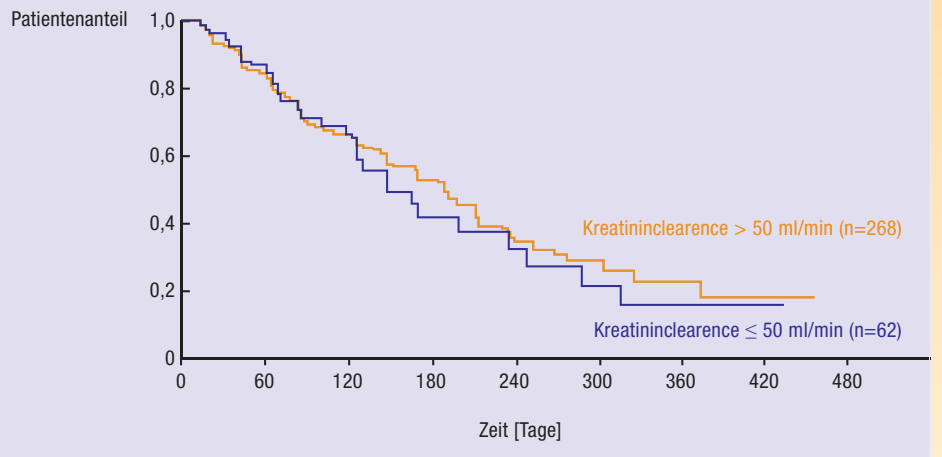

Abb. 2. Progressionsfreies Intervall in der APEX-Studie in Abhängigkeit von der Nierenfunktion [nach 4]. enz (Dialysepflicht) untersuchten ChananKhan et al. bei 24 Myelom-Patienten in einer retrospektiven Fall-Analyse [6]. Trotz der starken Nierenfunktionseinschränkungen konnte die Therapie über median 5 Zyklen gegeben werden. 83\% der Patienten erhielten den Proteasaminibitor in der empfohlenen Dosierung von $1,3 \mathrm{mg} / \mathrm{m}^{2}$ an den Tagen 1,4 , 8, 11, q3w. Die Art und Intensität der beobachteten Nebenwirkungen sowie das objektive Ansprechen waren mit den zuvor bei nicht-dialysepflichtigen Patienten beobachteten Behandlungsergebnissen vergleichbar. Darüber hinaus konnte nach schnellem Therapieansprechen bei einem Patienten sofort auf die Dialyse verzichtet werden, drei weitere wurden im Behandlungsverlauf von der Dialyse unabhängig.

Bestätigt wurden diese Daten durch die beim XI. International Myeloma Workshop in Kos/Griechenland vorgestellten Ergebnisse einer neuen Subgruppenanalyse der APEXStudie, in der die Wirksamkeit und Verträglichkeit von Bortezomib in Abhängigkeit von der Kreatinin-Clearance $(\mathrm{CrCl})$ analysiert wurden [4]. Bei Patienten mit einer $\mathrm{CrCl} \leq$ $50 \mathrm{ml} / \mathrm{min}$ zeigte sich ein vergleichbares Verträglichkeitsprofil und Therapieansprechen $(\mathrm{CR} / \mathrm{nCR}$, Zeit bis zur 1. Response) wie bei Patienten mit leichter Niereninsuffizienz oder nierengesunden Patienten. Das mediane progressionsfreie Intervall (4,9 vs. 6,2 Monate; $\mathrm{p}=0,62$, Abb. 2) und das mediane $\mathrm{Ge}$ samtüberleben (22,8 vs. 30,0 Monate; $\mathrm{p}=$ 0,07 ) waren ebenfalls nicht signifikant unterschiedlich. Wie im APEX-Gesamtkollektiv und bei Patienten mit einer $\mathrm{CrCl}>50 \mathrm{ml} / \mathrm{min}$ wurde auch bei Patienten mit mäßiger und schwerer Niereninsuffizienz $(\mathrm{CrCl} \leq 50 \mathrm{ml} / \mathrm{min})$ eine im Vergleich zu Dexamethason statistisch signifikante Verlängerung des progressionsfreien Intervalls beobachtet (4,9 vs. 2,8 Monate; $p=0,02$ ).

\section{Potenzial bei Skelettereignissen}

Circa 70-80\% der Myelom-Patienten entwickeln Osteolysen, die mit Schmerzen, pathologischen Frakturen und Hypercalcämie einhergehen und besonders bei Verlust der Autonomie zu einer starken Einschränkung der Lebensqualität führen. Ursächlich für diese Skelettereignisse beim multiplen Myelom ist nach In-vitro-Daten ein Circulus vitiosus, bei dem die Myelomzellen über die vermehrte Expression des Transkriptionsfaktors NFkappaB die Bildung und Aktivität der Osteoklasten fördern und $u$. a. über die Beeinflussung des Wingless-type (Wnt)-Signalwegs die Differenzierung und Funktion der Osteo- blasten hemmen. Dies kann wiederum zur vermehrten Proliferation, Migration und Invasion der Myelomzellen sowie zur Chemoresistenz führen (Abb. 3) [7, 8].

Die Behandlung mit Bisphosphonaten hemmt zwar die Osteoklasten-induzierte Knochenresorption, fördert aber nicht den Knochenaufbau durch die Osteoblasten. Hingegen kann der Proteasominhibitor Bortezomib nach den vorliegenden Daten offensichtlich beide Wege des Knochenstoffwechsels positiv beeinflussen, wie die Analyse spezifischer Surrogatmarker zeigte [9]. Unter anderem wurden in einer klinischen Studie mit $34 \mathrm{~Pa}$ tienten bereits nach 4 Zyklen Bortezomib eine signifikante Reduktion des Osteoblasteninhibitors DKK-1 sowie des Osteoklastenaktivators RANKL (Receptor Activator of NF-kappa B Ligand) beobachtet. Nach derzeitiger Studienlage ist die Hemmung der Osteoklasten und Aktivierung der Osteoblastenfunktion durch Bortezomib einmalig und wird bei anderen Anti-Myelomtherapien nicht beobachtet.

Dr. Barbara Bornkessel, Bremen

\section{Quelle}

«Aktuelle Entwicklungen in der Myelomtherapie mit Velcade ${ }^{\circledR}$ (Bortezomib): Hohe Ansprechqualität und verlängertes Überleben», Pressekonferenz im Rahmen des DGHO-Kongresses, Basel, 6. Oktober 2007, veranstaltet von Ortho Biotech, Division of Janssen-Cilag GmbH, Neuss.

\section{Literatur}

1 Richardson P, et al.: Blood First Edition Paper, prepublished online August 9, 2007; DOI 10 1182/blood-2006-08-036947.

2 Niesvizky R, et al : ASH 2006; Abstr \# 3529 , Posterpräsentation. 3 Richardson P, et al.: ASH 2005; Abstr \# 2547.

4 San-Miguel JF, et al.: International Myelom Workshop 2007, Abstr PO-1114, Posterpräsentation

5 Mulkerin D, et al.: ASCO 2006; Abstr \# 2032.

6 Chanan-Khan AA, et al.: Blood 2007;109:2604-2606.

7 Heider U, et al. Eur J Cancer 2006:42:1544-1553.

8 Terpos E, et al.: Blood 2007:110:1098-1104.

9 Terpos E, et al.: ASH 2006; Abstr \# 506.

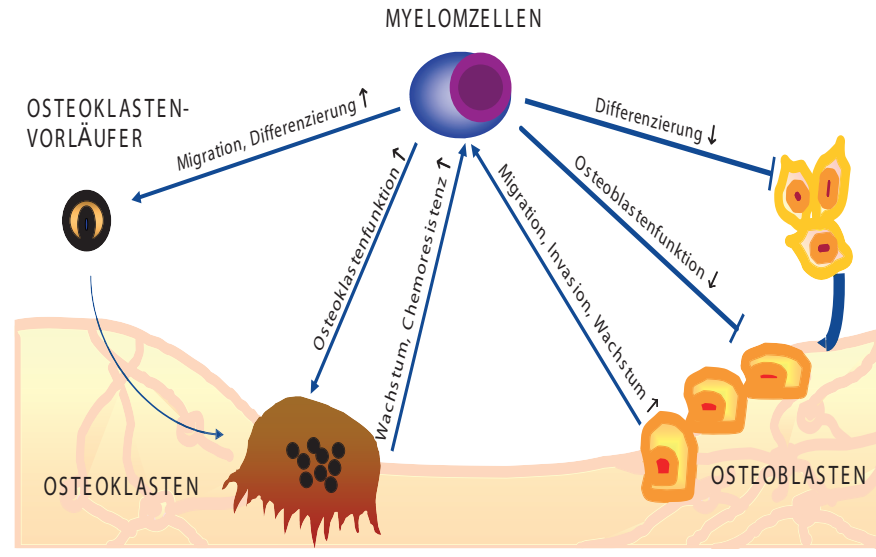

Abb. 3. Circulus vitiosus beim multiplen Myelom [modifiziert nach Sezer, Pressekonferenz im Rahmen des DGHO 2007].
Impressum

Aktuelle Entwicklungen in der Therapie des multiplen Myeloms

Bortezomib: Hohe Ansprechqualität

und verlängertes Überleben

PharmaForum in ONKoLogIe 30 | 12 | 07

C. 2007 by S. Karger Verlag für Medizin und Naturwissenschaften $\mathrm{GmbH}$ Lörracher Straße 16a

79115 Freiburg, Deu

Mit freundlicher Unterstützung von ORTHO BIOTECH, DIVISION OF JANSSEN-CILAG GmbH, Neuss.

Der Verlag und die Herausgeber der Zeitschrift übernehmen keine Verantwortung für diese Rubrik. 